\title{
Reduced injection current induced blueshift in an InGaN/GaN quantum- well light-emitting diode of prestrained growth
}

\author{
Chi-Feng Huang, Cheng-Yen Chen, Chih-Feng Lu, and C. C. Yang ${ }^{\text {a) }}$ \\ Graduate Institute of Electro-Optical Engineering, National Taiwan University, No. 1, Section 4, Roosevelt \\ Road, Taipei, Taiwan 10617, Republic of China \\ and Department of Electrical Engineering, National Taiwan University, No. 1, Section 4, Roosevelt Road, \\ Taipei, Taiwan 10617, Republic of China
}

(Received 17 June 2007; accepted 9 July 2007; published online 2 August 2007)

\begin{abstract}
The authors demonstrate the smaller blueshift in increasing injection current level of an InGaN/GaN quantum-well (QW) light-emitting diode (LED) of a longer electroluminescence (EL) peak wavelength based on the prestrained growth technique when compared with the result of a LED of a shorter EL peak wavelength based on the conventional growth technique. The smaller blueshift can be attributed to more contribution to light emission from the deeper QWs of higher indium contents when the injection current level is increased in the prestrain sample. It can also be attributed to the stronger carrier localization because of the stronger composition clustering in the prestrain sample of higher indium contents. Carrier localization can reduce the influences of the quantum-confined Stark effect and its screening process. () 2007 American Institute of Physics. [DOI: $10.1063 / 1.2767243$ ]
\end{abstract}

The quantum-confined Stark effect (QCSE) exists in InGaN/GaN quantum wells (QWs) due to the strong piezoelectric fields generated in the well layers. ${ }^{1-3}$ Such a piezoelectric field leads to the potential tilt resulting in the reduction of carrier recombination rate and the redshift of emission spectrum. In a light-emitting diode (LED) fabricated with an InGaN/GaN QW structure, when electric current is injected into the QWs, the carrier screening of the QCSE flattens the potential across a QW leading to a blueshift of LED output spectrum. The blueshift becomes more significant in a QW structure of higher indium content, i.e., in a LED for emitting longer-wavelength photons (green, yellow, and red), because of the stronger piezoelectric field in a QW of a larger lattice mismatch. Such a blueshift behavior represents one of the unsolved problems in fabricating high-quality InGaN-based LEDs.

Recently, this research group has developed a prestrained growth technique for enhancing indium incorporation in an InGaN/GaN QW such that efficient yelloworange-red and white-light LEDs can be fabricated. ${ }^{4-6}$ Basically, in the prestrained growth technique, we grow a low indium $\mathrm{InGaN} / \mathrm{GaN}$ QW before the designated lightemitting high indium InGaN/GaN QWs. With the low indium well layer, the GaN barrier layer right above it is tensile strained such that the indium incorporation during the growth of the subsequent QWs becomes easier. ${ }^{7,8}$ Hence, their indium contents can be significantly increased, leading to the efficient emissions of yellow, orange, and red colors. In this situation, the indium contents among the high indium QWs are nonuniform. The high indium QWs (deeper QWs) closer to the low indium QW receive stronger prestrain effects and hence have relatively higher indium contents. Because hole migration is much slower than that of electron in nitride compounds, holes are mainly distributed in the shallower QWs (close to the $p$-type layer) at low injection current levels. ${ }^{9}$ When the injection current level increases, a

${ }^{a)}$ FAX: 886-2-23652637; electronic mail: ccy@cc.ee.ntu.edu.tw higher density of holes can reach the deeper QWs and contribute to longer-wavelength light. ${ }^{10,11}$

In this letter, we report and explain the smaller blueshift of the LED fabricated with an InGaN/GaN QW structure of prestrained growth at large injection current when compared with a control (unprestrained) sample. It is found that the QCSE under the prestrained growth condition is actually enhanced. The smaller blueshift in the prestrain sample is attributed to the injection current dependence of hole distribution among QWs and the enhanced carrier localization in the QWs of increased indium contents.

Both the control and prestrain samples were grown with metal-organic chemical vapor deposition. First, a $30 \mathrm{~nm} \mathrm{GaN}$ nucleation layer was grown on $c$-plane sapphire substrate at $530{ }^{\circ} \mathrm{C}$ in the control sample. Then, after the growth of a $2 \mu \mathrm{m} n$-GaN at $1080^{\circ} \mathrm{C}$, five periods of $\mathrm{InGaN} / \mathrm{GaN} \mathrm{QW}$ with $3 \mathrm{~nm}$ in the well thickness and $15 \mathrm{~nm}$ in the barrier thickness were deposited. In growing the QWs, after the growth of a $3 \mathrm{~nm}$ InGaN QW layer at $670{ }^{\circ} \mathrm{C}$, the growth temperature was ramped to $870{ }^{\circ} \mathrm{C}$ within $1 \mathrm{~min}$ for depositing the GaN barrier. After the growth of the QWs, a $30 \mathrm{~nm}$ $p-\mathrm{Al}_{0.2} \mathrm{Ga}_{0.8} \mathrm{~N}$ layer, followed by a $120 \mathrm{~nm} p$-GaN layer (both grown at $945^{\circ} \mathrm{C}$ ) were deposited. In the prestrain sample, the LED structure is the same as that of the control sample except that an extra $\mathrm{InGaN} / \mathrm{GaN} \mathrm{QW}$ of a lower indium content (grown at $750{ }^{\circ} \mathrm{C}$ ) was deposited between the $n$-GaN layer and the five high indium QWs of the same growth conditions.

Figure 1 shows the normalized photoluminescence (PL) and electroluminescence (EL) spectra of the two samples. The EL spectra were obtained at $20 \mathrm{~mA}$ in injection current. One can see that the PL spectral peak of the prestrain sample (at $572.7 \mathrm{~nm}$ ) is only slightly redshifted from that of the control sample (at $568.7 \mathrm{~nm}$ ). However, the EL spectral peak of the prestrain sample (at $589.7 \mathrm{~nm}$ ) is significantly redshifted by $18.1 \mathrm{~nm}$ from that of the control sample (at $571.6 \mathrm{~nm}$ ). It is noted that throughout this letter, the spectral peaks are obtained after filtering out the Fabry-Perot oscillations in the spectra. The significant difference between the 


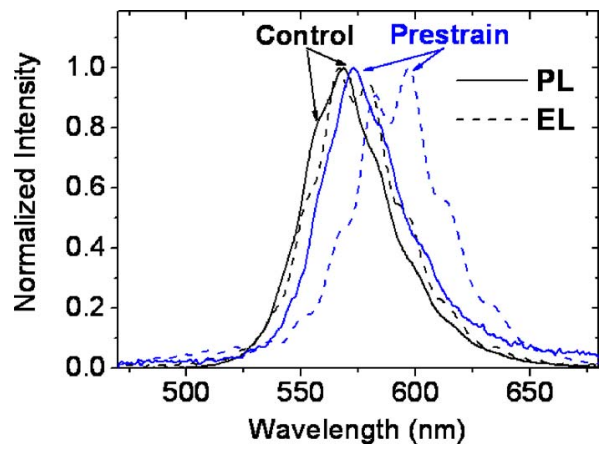

FIG. 1. (Color online) Normalized PL and EL spectra of the control and prestrain samples.

PL and EL spectral peaks of the prestrain sample manifests the unique feature of the prestrained growth. ${ }^{4,6}$ It is attributed to the nonuniform indium-content distribution among the five high indium QWs. The deeper QWs have higher indium contents and hence emit longer-wavelength light. Since PL spectrum originates mainly from the shallower QWs and EL spectrum has more contribution from the deeper QWs, the EL spectrum is significantly redshifted from the PL spectrum in the prestrain sample. Because the prestrain effects are weak on the shallower QWs, the indium contents of the shallower QWs in the prestrain sample are about the same as those of the QWs in the control sample, ${ }^{8}$ the two PL spectra are not much different, as shown in Fig. 1.

Figure 2 shows the EL spectra of the control sample at various injection current levels. The inset shows the spectral peak variations with injection current. Here, one can see that the spectral peak blueshifts by $15.6 \mathrm{~nm}$ (from $580.6 \mathrm{~nm}$ at $3 \mathrm{~mA}$ to $565 \mathrm{~nm}$ at $50 \mathrm{~mA}$ ) when the injection current increases from 3 to $50 \mathrm{~mA}$. Figure 3 shows the counterparts of Fig. 2 in the prestrain sample. Here, one can see that the spectral peak blueshifts by $9.1 \mathrm{~nm}$ (from $592.8 \mathrm{~nm}$ at $7 \mathrm{~mA}$ to $583.7 \mathrm{~nm}$ at $50 \mathrm{~mA}$ ). The slight redshift of spectral peak in increasing injection current when it is smaller than $7 \mathrm{~mA}$ is attributed to the increased hole density in the deeper QWs, which have higher indium contents and emit longerwavelength light. Therefore, in this injection current range, as the current level increases, the EL spectrum tends to redshift until the hole density distribution reaches a saturated condition, beyond which the screening of the QCSE starts to play the major role in spectral shift. Nevertheless, the blueshift of only $9.1 \mathrm{~nm}$ when the injection current increases from 7 to $50 \mathrm{~mA}$ in the prestrain sample is significantly

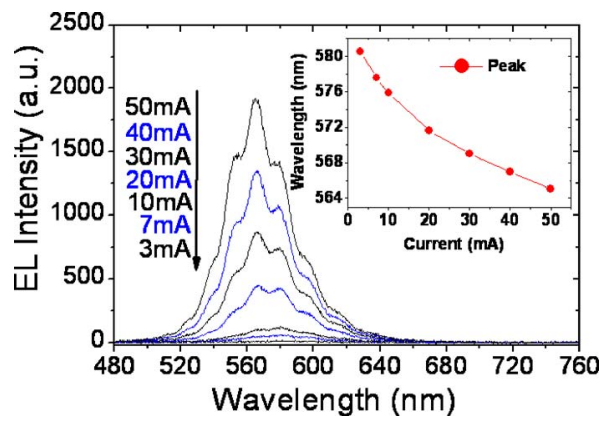

FIG. 2. (Color online) EL spectra of the control sample at various injection current levels. The inset shows the spectral peak variation with the injection current.

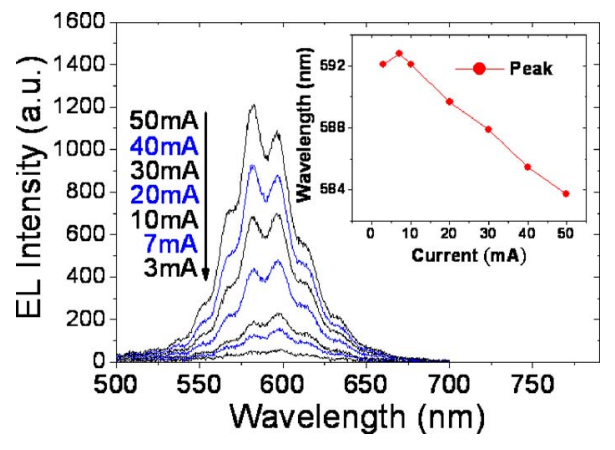

FIG. 3. (Color online) EL spectra of the prestrain sample at various injection current levels. The inset shows the spectral peak variation with the injection current.

smaller than that of $12.6 \mathrm{~nm}$ of the control sample in the same injection current range.

Figures 4 and 5 show the PL spectra of the control and prestrain samples, respectively, at various reverse-biased voltages. The insets show the spectral peak variations with the reverse-biased voltage of the individual samples. Because the piezoelectric field is along the direction from the $p$-type layer toward the $n$-type layer and dominates the built-in electric field, the reverse-biased voltage tends to cancel the piezoelectric field. ${ }^{12}$ Hence, by applying a reversebiased voltage, an InGaN/GaN QW LED sample of a stronger QCSE is expected to show a more significant blueshift in PL spectrum. In Fig. 4, one can see that the blueshift of PL spectral peak in changing the reverse-biased voltage from 0 to $12 \mathrm{~V}$ is only $11.3 \mathrm{~nm}$ (from $565.8 \mathrm{~nm}$ at $0 \mathrm{~V}$ to $554.5 \mathrm{~nm}$ at $-12 \mathrm{~V}$ ) in the control sample. On the other hand, in the prestrain sample, the blueshift is as large as $30.8 \mathrm{~nm}$ (from $572.5 \mathrm{~nm}$ at $0 \mathrm{~V}$ to $541.7 \mathrm{~nm}$ at $-12 \mathrm{~V}$ ) in the same reverse voltage range. Also, the PL intensity variation in varying the reverse voltage of the prestrain sample is significantly smaller than that of the control sample. Those observations imply that the QCSE in the prestrain sample is significantly stronger than that in the control sample. Such a conclusion can be attributed to the higher indium contents and hence the larger lattice mismatch between wells and barriers in the prestrain sample.

The smaller EL blueshift of the prestrain sample in increasing the injection current seems to be in contradiction to the observation of the stronger QCSE in this sample. The smaller EL blueshift caused by the QCSE screening in the prestrain sample can be attributed to two possible mechanisms. First, as mentioned before, the deeper QWs in the

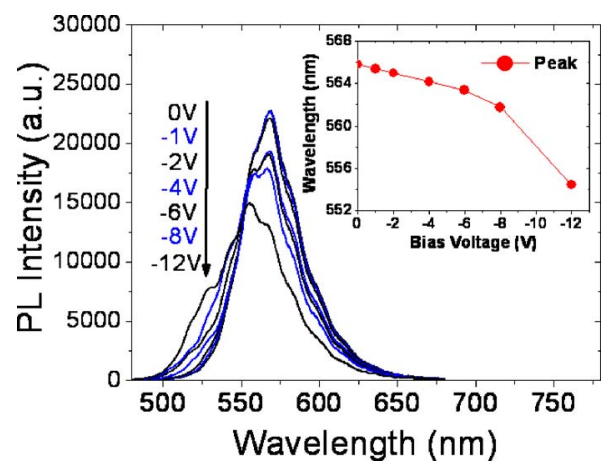

FIG. 4. (Color online) PL spectra of the control sample at various reversebiased voltages. The inset shows the spectral peak variation with the

reverse-biased voltage.
to AIP license or copyright; see http://apl.aip.org/apl/copyright.jsp 


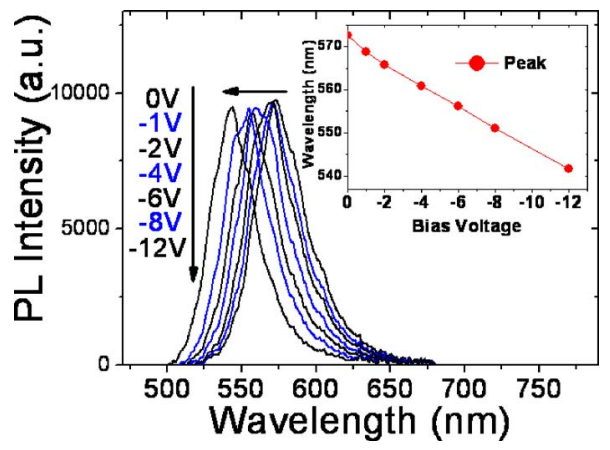

FIG. 5. (Color online) PL spectra of the prestrain sample at various reversebiased voltages. The inset shows the spectral peak variation with the reverse-biased voltage.

prestrain sample have higher indium contents and hence emit photons of longer wavelengths. As the injection current increases, the hole densities in the deeper QWs increase and hence the LED emitting wavelength tends to redshift for balancing the blueshift caused by the QCSE screening. Second, the composition clustering in the deeper QWs of higher indium contents is expected to be stronger than that in the control sample. ${ }^{13}$ In this situation, the stronger carrier localization guarantees the overlap of electron and hole wave functions and reduces the influences of the QCSE and its screening effect. ${ }^{14}$ Either mechanism is useful for developing long-wavelength visible LEDs based on InGaN/GaN QWs.

In summary, we have demonstrated the smaller blueshift in increasing injection current level in an $\mathrm{InGaN} / \mathrm{GaN} \mathrm{QW}$ LED of a longer EL peak wavelength based on the prestrained growth technique when compared with the result of a LED of a shorter EL peak wavelength based on the conventional growth technique. This behavior could be attributed to more contribution to light emission from the deeper QWs of higher indium contents when the injection current level was increased in the prestrain sample. It could also be attributed to the stronger carrier localization because of the stronger composition clustering in the prestrain sample of higher indium contents. Carrier localization can reduce the influences of the QCSE and its screening effect.

This research was supported by National Science Council, The Republic of China under Grant Nos. NSC 95-2120M-002-012 and NSC 95-2221-E-002-287 and by US Air Force Scientific Research Office under Contract No. AOARD-06-4052.

${ }^{1}$ S. F. Chichibu, A. C. Abare, M. S. Minsky, S. Keller, S. B. Fleischer, J. E. Bowers, E. Hu, U. K. Mishra, L. A. Coldren, S. P. DenBaars, and T. Sota, Appl. Phys. Lett. 73, 2006 (1998).

${ }^{2}$ P. Riblet, H. Hirayama, A. Kinoshita, A. Hirata, T. Sugano, and Y. Aoyagi, Appl. Phys. Lett. 75, 2241 (1999).

${ }^{3}$ E. Berkowicz, D. Gershoni, G. Bahir, E. Lakin, D. Shilo, E. Zolotoyabko, A. C. Abare, S. P. Denbaars, and L. A. Coldren, Phys. Rev. B 61, 10994 (2000).

${ }^{4}$ C. F. Huang, T. Y. Tang, J. J. Huang, W. Y. Shiao, C. C. Yang, C. W. Hsu, and L. C. Chen, Appl. Phys. Lett. 89, 051913 (2006).

${ }^{5}$ H. S. Chen, C. F. Lu, D. M. Yeh, C. F. Huang, J. J. Huang, and C. C. Yang, IEEE Photonics Technol. Lett. 18, 2269 (2006).

${ }^{6}$ C. F. Huang, T. Y. Tang, J. J. Huang, and C. C. Yang, Appl. Phys. Lett. 90, 151122 (2007)

${ }^{7}$ Y. S. Chen, L. J. Yao, Y. L. Lin, L. Hung, C. F. Huang, T. Y. Tang, J. J. Huang, W. Y. Shiao, and C. C. Yang, J. Cryst. Growth 297, 66 (2006).

${ }^{8}$ W. Y. Shiao, C. F. Huang, T. Y. Tang, J. J. Huang, and C. C. Yang, J. Appl. Phys. 101, 113503 (2007).

${ }^{9}$ S. Fujita, M. Funato, D. C. Park, Y. Ikenaga, and S. Fujita, MRS Internet J. Nitride Semicond. Res. 4S1, G6.31 (1999).

${ }^{10}$ H. S. Chen, D. M. Yeh, C. F. Lu, C. F. Huang, J. J. Huang, and C. C. Yang, Appl. Phys. Lett. 89, 093501 (2006).

${ }^{11}$ C. F. Lu, D. M. Yeh, H. S. Chen, C. F. Huang, J. J. Huang, and C. C. Yang, IEEE Photonics Technol. Lett. 18, 2671 (2006).

${ }^{12}$ T. Takeuchi, C. Wetzel, S. Yamaguchi, H. Sakai, H. Amano, I. Akasaki, Y. Kaneko, S. Nakagawa, Y. Yamaoka, and N. Yamada, Appl. Phys. Lett. 73, 1691 (1998).

${ }^{13}$ Y. S. Lin, K. J. Ma, C. Hsu, S. W. Feng, Y. C. Cheng, C. C. Liao, C. C. Yang, C. C. Chuo, C. M. Lee, and J. I. Chyi, Appl. Phys. Lett. 77, 2988 (2000).

${ }^{14}$ Y. C. Cheng, E. C. Lin, C. M. Wu, C. C. Yang, J. R. Yang, A. Rosenauer, K. J. Ma, S. C. Shi, L. C. Chen, C. C. Pan, and J. I. Chyi, Appl. Phys. Lett. 84, 2506 (2004). 